\title{
Linking microbial population succession and DOM molecular transformation in Synechococcus- derived organic matter addition incubations
}

\author{
YU WANG1, RUI XIE2, QI CHEN3, RUANHONG CAI4,
} CHEN He5, WeIDONG GUO6, QUAN ShI7, NiANZHI JIAO8*, QIANG ZHENG9*

1 Xiamen Uniersity, Xiamen, China (wangyu@xmu.edu.cn) 2 Xiamen Uniersity, Xiamen, China (xierui@stu.xmu.edu.cn) 3 Xiamen Uniersity, Xiamen, China (qichen@stu.xmu.edu.cn)

4 Xiamen Uniersity, Xiamen, China (crh1987@163.com)

5 China University of Geosciences, Beijing, China (hechen1028@126.com)

6 Xiamen Uniersity, Xiamen, China (wdguo@xmu.edu.cn)

7 China University of Geosciences, Beijing, China (geochem@vip.sina.com)

8 Xiamen Uniersity, Xiamen, China (*correspondence: jiao@xmu.edu.cn)

9 Xiamen Uniersity, Xiamen, China (*correspondence: zhengqiang@xmu.edu.cn)

Phytoplankton are major source of labile dissolved organic matter (DOM) in the upper ocean which fuels heterotrophic bacterial metabolic activity. However, we still lack the knowledge on the mechanism underlying the DOM transformation by the microorganisms at chemical molecular level. Here we study on how picocyanobacteria-derived DOM was transformed by successive coastal microorganism metabolism during 180-day incubations and their implication for ecosystem functioning. Associations between total/active microbial communities and DOM molecules were analyzed. The results show that compositions of both microbes and DOM molecules trend to be homogeneous over the incubations. The utilization/degradation of DOM strongly associates with the bioavailable $\mathrm{N}$-containing organic molecules and the transformation of different forms of inorganic nitrogen nutrients. The $\mathrm{N}$ - and S-containing organic molecules decreased their $\mathrm{m} / \mathrm{z}$ values over the incubations, suggesting their different fates via microbial degradation. The SEM model revealed that the active microbial composition was directly influenced by labile DOM and semi-labile DOM, and played more important roles in shaping the DOM molecular composition and refactory DOM production compared to total microbial composition. This study sheds light on the interactions between marine microbes and organic molecules, and advances our understanding of microbial processing DOM pool in the ocean. 